\title{
E-cadherina: expresión molecular en carcinomas de estómago de tipo difuso y tipo intestinal. Estudio realizado en Lima, Perú
}

\author{
F. Arévalo, E. Monge ${ }^{1}$ y D. Morales ${ }^{1}$ \\ Servicio de Anatomía Patológica. Hospital Nacional Dos de Mayo. Universidad Nacional Mayor de San Marcos \\ ${ }^{\prime}$ Servicio de Gastroenterología Daniel Alcides Carrión. Universidad Nacional Mayor de San Marcos. Lima, Perú
}

\section{RESUMEN}

Objetivo: según la clasificación de Lauren el adenocarcinoma tipo difuso es conocido por tener un comportamiento más agresivo e infiltrante que el adenocarcinoma tipo intestinal. Desde hace una década, diferentes estudios han postulado que esta cualidad del adenocarcinoma tipo difuso se debe en parte a la pérdida de expresión de la molécula E-cadherina, proteína de la membrana celular cuya función es permitir la adhesión intercelular.

Métodos: el presente trabajo es un estudio transversal comparativo que mide la expresión de la proteína de adhesión intercelular E-cadherina con la ayuda de inmunohistoquímica, seleccionando aleatoriamente 10 casos de carcinoma de células en anillo de sello y 10 casos de carcinoma tipo intestinal, comparándolos.

Resultados: encontramos una mayor expresión de E-cadherina en los adenocarcinomas de tipo intestinal (100\%) que en los carcinomas de células en anillo de sello (40\%) siendo esta diferencia estadísticamente significativa usando el test de Fisher $(\mathrm{p}=$ 0,011). La mayor pérdida de E-cadherina permitiría entender la mayor capacidad infiltrante del adenocarcinoma tipo difuso respecto al adenocarcinoma tipo intestinal.

Palabras clave: Carcinoma de células en anillo de sello gástrico. Adenocarcinoma gástrico. E-cadherina.

\begin{abstract}
Objective: signet ring carcinoma of the stomach is well known to be more aggressive and infiltrating than adenocarcinoma. Different studies have proposed that signet ring cell carcinoma would be more infiltrating because of the lost of E-cadherin expression, this cadherin is a class of protein cell membrane protein which plays an important role in cell-adhesion.

Methods: we carried out a transversal comparative study, in order to measure the E-cadherin expression in 10 cases of signet ring cell and in 10 cases of adenocarcinoma, with help of immunohistochemistry.

Results: we found a bigger expression of E-cadherin in adenocarcinomas (100\%) than signet ring cell carcinoma (40\%), this difference was significative using Fisher test $(p=0,011)$. The lost of E-cadherine would explain the bigger infiltrating capacity in comparison to adenocarcinoma.
\end{abstract}

Key words: Gastric signet ring cell carcinoma. Gastric adenocarcinoma. E-cadherine.

Arévalo F, Monge E, Morales D. E-cadherina: expresión molecular en carcinomas de estómago de tipo difuso y tipo intestinal. Estudio realizado en Lima, Perú. Rev Esp Enferm Dig 2007; 99: 325-329.

\section{INTRODUCCIÓN}

Desde que Lauren describiera en 1965 dos tipos de carcinoma gástrico con pronóstico y comportamiento

Recibido: 24-01-07.

Aceptado: 19-04-07.

Correspondencia: Fernando Arévalo. Servicio de Anatomía Patológica. Hospital Nacional Dos de Mayo. Universidad Nacional Mayor de San Marcos. Lima, Perú. e-mail: fer1301@hotmail.com biológico diferentes, el tipo intestinal y el tipo difuso (1), las diferencias entre ambos tipos de carcinoma gástrico no sólo han sido muy notorias desde el punto de vista morfológico sino que también han sido muy estudiadas y conocidas desde el punto de vista clínico y epidemiológico. Desde el punto de vista morfológico el adenocarcinoma tipo intestinal está formado por estructuras glandulares muy cohesivas, y se corresponde con los adenocarcinomas tubular y papilar de la clasificación de la OMS, mientras que el adenocarcinoma de tipo difuso 
está compuesto por células individuales y dispersas y se corresponde con el carcinoma de células en anillo de sello de la clasificación de la OMS; asimismo, desde el punto de vista clínico y epidemiológico existen muchos trabajos que demuestran que el adenocarcinoma tipo difuso es más frecuente en mujeres, en gente joven y su comportamiento biológico es de tipo infiltrante y de localización principalmente en fondo gástrico, mientras que el adenocarcinoma tipo intestinal se presenta con más frecuencia en varones, adultos mayores, siendo su presentación de tipo exofítico y de localización principalmente antral (2-4), por otro lado la asociación con Helicobacter pylori y lesiones precancerosas ha sido comprobada con el carcinoma de tipo intestinal, mientras que esta asociación no parece ser tan evidente con el adenocarcinoma tipo difuso $(5,6)$. No obstante, si las diferencias clínicas están bien establecidas, poco se conoce de las diferencias a nivel molecular entre estos dos tipos de carcinoma. Algunos estudios señalan que el adenocarcinoma tipo intestinal a diferencia del difuso, presenta mayor expresión de receptores de crecimiento epidérmico erb B2 y erb B3 (7), así como de proteínas reguladoras de apoptosis como bcl-2 y BAX, hallazgos que nos hablan de las características replicativas de los carcinomas pero no de su capacidad invasiva. En 1994, Becker y cols. demostró que los carcinomas de células en anillo de sello o adenocarcinomas tipo difuso para la clasificación de Lauren, presentan con más frecuencia mutaciones en el gen de la E-cadherina que el resto de adenocarcinomas (8), desde esa publicación, se han realizado algunos trabajos referentes a la presencia de E-cadherina con resultados divergentes, no conocemos de ningún trabajo parecido realizado en Perú. El presente trabajo analiza características clínico histológicas de los casos de adenocarcinoma tipo difuso y tipo intestinal diagnosticados en el periodo 2000-2005 y mide la expresión de la proteína de adhesión intercelular E-cadherina en ambos grupos.

\section{MATERIALES Y MÉTODOS}

El presente es un trabajo transversal comparativo. Se identificaron todos los casos diagnosticados como adenocarcinoma tipo difuso y adenocarcinoma tipo intestinal de estómago, durante el periodo 2000-2005 en muestras obtenidas mediante biopsia endoscópica; se definió como caso de adenocarcinoma tipo difuso a aquel tumor constituido por más del $50 \%$ de su población celular por grupos de estas células en "anillo de sello" (9), y como caso de adenocarcinoma tipo intestinal a aquellos que presentaran grupos de células neoplásicas formando estructuras glandulares cohesivas. Para el estudio de la molécula E-cadherina se tomó una muestra aleatoria de cada grupo, de 10 casos cada uno, con la ayuda del programa SPSS $^{\circledast}$ 13.0. Para la detección de E-cadherina fueron empleados anticuerpos monoclonales de la casa Zymed, compañía transnacional de laboratorios dedicados a la producción e investigación de anticuerpos para uso diagnóstico. Para la detección de E-cadherina fueron empleados anticuerpos monoclonales y fueron procesados mediante la técnica de inmunoperoxidasa, técnica que será descrita brevemente: se hacen los cortes con el microtomo, los microcortes se recogen con láminas sialinizadas, los mismos se desparafinan en la estufa y luego con xilol, posteriormente se rehidratan con alcohol y agua. La recuperación de los antígenos "escondidos por efecto del formol" se ejecuta mediante el método térmico, posteriormente se bloquea la peroxidasa endógena aplicando sobre el tejido agua oxigenada al 3\% por aproximadamente 10 minutos. Una vez recuperados los antígenos, se usa el anticuerpo primario, se incuba a temperatura ambiente por 30-60 minutos, luego el anticuerpo secundario biotinilado se incuba por otros 30 minutos, se aplica la streptavidina peroxidasa y finalmente el cromógeno diaminobenzidina.

Se consideró como célula neoplásica positiva para E-cadherina a aquella que presentase coloración dorada en la membrana celular luego del procedimiento inmunohistoquímico, debiendo ser la coloración clara e intensa, y como caso positivo fue considerado a aquel que tuviese más del $60 \%$ de la población neoplásica positivo para E-cadherina, y como caso negativo a aquel que tuviese más del $40 \%$ de la población neoplásica negativo para E-cadherina. Para determinar la significancia estadística de la diferencia entre ambos grupos, se usó la prueba de Chi cuadrado de Pearson, con corrección de Fisher debido al tamaño de la muestra estudiada, con ayuda del programa SPSS ${ }^{\circledR}$ 13.0. También se consignó y midió la frecuencia de algunas variables como sexo y edad.

\section{RESULTADOS}

Se identificaron 90 casos de adenocarcinoma tipo difuso, 56 varones $(62,5 \%)$ y 34 mujeres $(37,5 \%)$, con edad media de 55,9 años y 382 casos de adenocarcinoma tipo intestinal: 248 varones $(64,9 \%)$ y 134 mujeres $(35 \%)$ con edad media de 64,2 años.

Se realizó un cuadro de contingencia para comparar la positividad de E-cadherina en los adenocarcinoma intestinales y entre los adenocarcinoma difuso encontrándose un $100 \%$ de positividad en los adenocarcinomas intestinales y un $40 \%$ de positividad en los carcinomas de células en anillo de sello, resultando esta diferencia significativa, usando el test de Chi cuadrado de Pearson, con corrección de Fisher $\mathrm{p}=0,011$.

\section{DISCUSIÓN}

El control de la adhesión y locomoción celular son blancos fundamentales en la progresión de todo tumor. Cuando un tumor es más invasivo es porque ha perdido su capaci- 

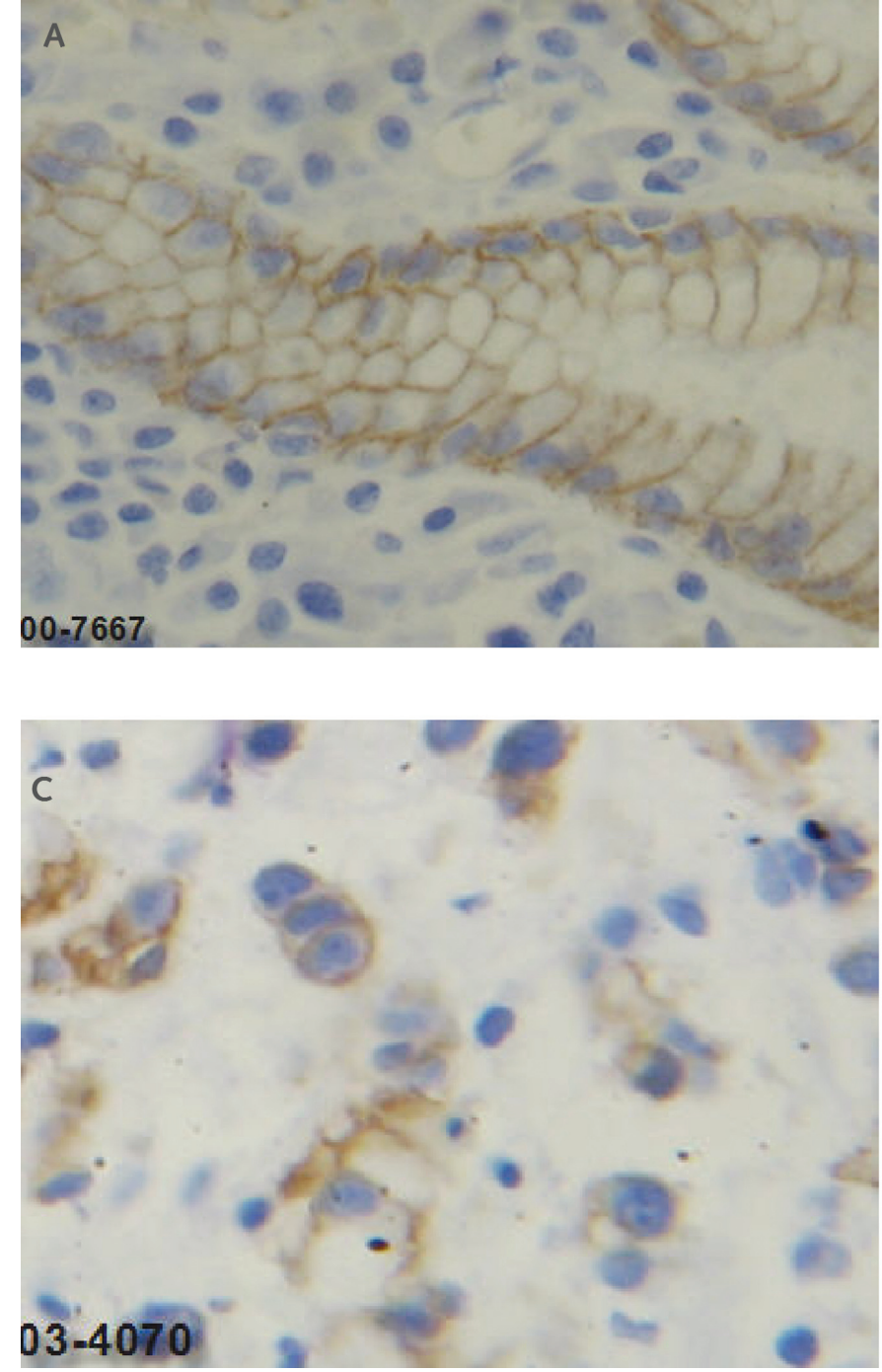
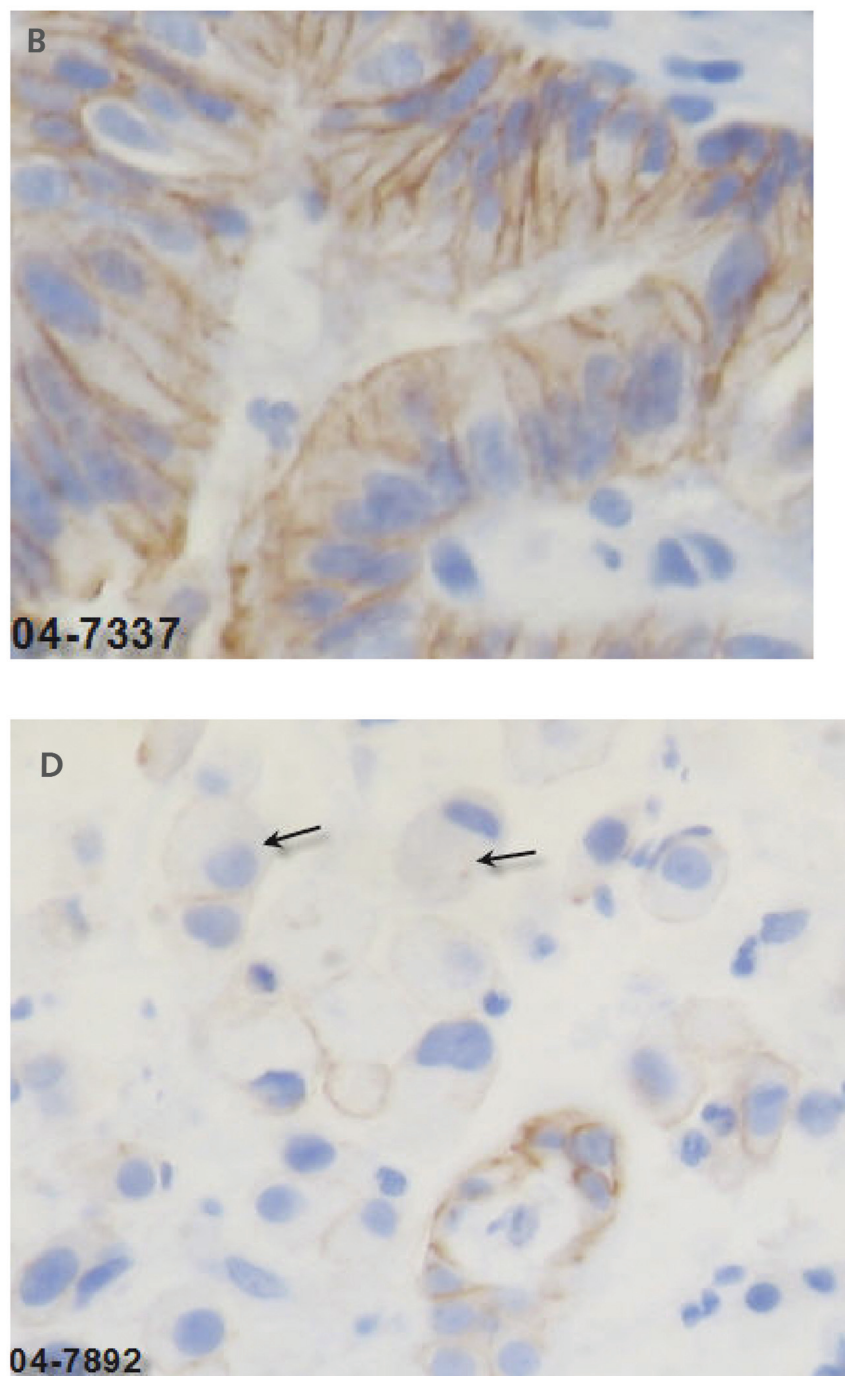

04-7892

Fig. 1. A. Imagen de una glándula normal marcada inmunohistoquímicamente para E-cadherina, obsérvese la coloración dorada de la membrana celular. B. Imagen de un adenocarcinoma positivo para E-cadherina. C. Carcinoma de céñulas en anillo de sello con positividad para E-cadherina. D. Microfotografía que muestra las tradicionales células en anillo de sello negativas para E-cadherina. Inmunoperoxidasa 200X.

dad de adhesión intercelular; la glicoproteína de superficie E-cadherina es una de las moléculas más importantes que hacen posible la adhesión intercelular en los tejidos de estirpe epitelial. La E-cadherina es una glicoproteína, de 120 $\mathrm{Kd}$, calcio dependiente (10). Esta molécula se localiza en la superficie celular de las células epiteliales, en las regiones de contacto celular. La E-cadherina posee un pequeño dominio intracelular citoplasmático, el cual interactúa con la actina citoplasmática a través de unas moléculas de unión llamadas alfa, beta y gamma cateninas (11), gracias a esta propiedad, funciona como pieza clave en la adhesividad y cohesividad del tejido epitelial.

Por tanto, se puede suponer que la falta de expresión de esta molécula de adhesión permitiría en los adenocarcinomas un comportamiento más infiltrante; en los adenocarcinomas de estómago, el adenocarcinoma tipo difu- so es conocido clásicamente por ser más infiltrante y agresivo que los adenocarcinomas tipo intestinal, entonces se puede suponer que los adenocarcinomas tipo difuso tendrían una menor expresión de esta molécula de adhesión. Respecto a esta interrogante se han realizado trabajos con resultados divergentes. En 2002, en Corea, se estudió la expresión de E-cadherina en cáncer colónico; el 100\% de casos de carcinoma de células en anillo de sello fueron negativos para esta molécula y sólo $23 \%$ de los casos de adenocarcinomas bien diferenciados fueron negativos. Esta diferencia fue estadísticamente significativa, y demostró que los carcinomas en anillo de sello en colon tienden a perder esta molécula de unión intercelular (12), pero en el carcinoma gástrico tipo difuso o de células en anillo de sello, los trabajos realizados sobre la pérdida de esta molécula han tenido diferentes resultados, 
así por ejemplo, Chu y cols., en el 2002 estudiaron inmunohistoquímicamente, carcinomas de células en anillo de sello de estómago, de los cuales $43 \%$ de los casos mostraron ausencia de expresión para E-cadherina (13). Zhang y cols. encontraron positividad de E-cadherina en $56 \%$ de todos los casos de adenocarcinoma gástrico estudiados, pero no halló diferencia significativa entre los adenocarcinomas tipo intestinal y difuso (14). Por otro lado Chen y cols. encontraron una diferencia significativa entre la falta de expresión de cadherina en adenocarcinoma tipo difuso $(65 \%)$ en relación a los adenocarcinomas tipo intestinal (20\%) (15). En el presente trabajo encontramos que $40 \%$ de los casos de adenocarcinoma tipo difuso fueron positivos inmunohistoquímicamente para E-cadherina, mientras que todos los casos de adenocarcinomas tipo intestinal, fueron considerados como positivos para E-cadherina, siendo esta diferencia estadísticamente significativa.

La diferencia entre los resultados de diferentes autores y el nuestro debe realizarse teniendo en cuenta que la positividad o negatividad inmunohistoquímica se realiza arbitrariamente basándose en criterios previamente establecidos por el investigador; por ejemplo nosotros consideramos como caso negativo a aquel que tuviese más del $40 \%$ de la población neoplásica negativa para E-cadherina, pudiendo encontrar en estos casos pequeñas poblaciones que conservaban la expresión de E-cadherina; por lo que un punto común en lo reportado por otros investigadores y en este trabajo es que la ausencia de expresión de E-cadherina no se da en todos los casos de adenocarcinomas tipo difusos; incluso en un mismo caso de adenocarcinoma tipo difuso considerado como negativo para E-cadherina, puede encontrarse pequeños grupos de células que conservan la positividad, no existiendo diferencia con los casos de adenocarcinoma tipo intestinal en cuanto la calidad e intensidad de la inmunomarcación; además debemos señalar que en casos de adenocarcinomas tipo intestinal, hallamos pequeños grupos celulares de mayor indiferenciación que no marcaron para E-cadherina. Zhou y cols., en un estudio realizado en China, encontraron una relación inversa entre el grado de diferenciación tumoral y la expresión de E-cadherina, de esta manera encontraron los siguientes porcentajes de alteración de expresión de E-cadherina, 18,8\% en los adenocarcinomas bien diferenciados tipo papilar y $100 \%$ en los carcinomas indiferenciados (16); ante estos hallazgos, creemos que la ausencia en la expresión de E-cadherina estaría en relación a una pérdida de esta molécula durante la progresión tumoral, es decir, sería una característica de la indiferenciación neoplásica, dicha pérdida de E-cadherina se observaría principalmente en adenocarcinomas difusos, pero también en grupos indiferenciados de adenocarcinomas tipo intestinal. Sin embargo también aceptamos que existen casos que llevan una determinada precondición genética, como sucede en los carcinomas gástricos tipo difuso, con agregación familiar, en los cuales se han identificado mutaciones germinales inactiva- dores en el gen de la E-cadherina: CDH1, en familias neozelandesas y europeas $(17,18)$, o más recientemente alteraciones en la región promotora de ese gen en familias mexicanas, con predisposición conocida para desarrollar cáncer gástrico (19); no obstante consideramos que estos casos representan la minoría del total de casos de adenocarcinomas gástricos.

Algunos autores han reportado en lesiones consideradas como preneoplásicas, pérdida de expresión de E-cadherina; creemos que si la pérdida de E-cadherina fuese un evento temprano frecuente, entonces debe encontrarse con más frecuencia ausencia de E-cadherina en adenocarcinomas tipo intestinal, que son el siguiente eslabón en la secuencia carcinogénica: metaplasia, displasia y adenocarcinoma tipo intestinal, pero eso no fue lo encontrado, sin embargo consideramos que los casos en que se ha observado ausencia de E-cadherina en lesiones iniciales, podrían reflejar el reemplazo de la expresión de E-cadherina por Li-cadherina, molécula de adhesión que sólo se encuentra en el hígado e intestino delgado y ausente en estómago, y cuya presencia ha sido reportada en metaplasia intestinal y adenocarcinomas tipo intestinal gástrico, por un trabajo alemán (20). Este cambio de E-cadherina por Li-cadherina sería un rasgo diferenciación intestinal temprana en los adenocarcinomas, lo cual permitiría entender por qué algunos casos de adenocarcinomas morfológicamente cohesivos, con una aparente adhesividad conservada, resulten negativos inmunohistoquímicamente para E-cadherina.

Con referencia a la relación entre E-cadherina y la aparición de metástasis, Zhou y cols. encontraron una relación entre el tamaño y apariencia macroscópica del tumor y la ausencia de expresión de E-cadherina, pero no pudieron demostrar asociación con la profundidad de infiltración y tampoco con metástasis ganglionar. Similares resultados fueron obtenidos por un trabajo realizado en Chile (21); evidentemente la sola presencia de E-cadherina no garantiza la adhesividad intercelular, deben evaluarse otros factores de adhesión como las diferentes cateninas, por lo que puede ocurrir que un caso muy infiltrante y agresivo, sea inmunohistoquímicamente positivo para E-cadherina pero presente alteraciones en algunas de las cateninas; además debemos considerar la posibilidad que un caso de carcinoma infiltrante e inmunohistoquímicamente positivo para E-cadherina, exprese una E-cadherina no funcional, como lo planteado por Becker (22), quien usando un anticuerpo monoclonal, designado E-cad.delta 9-1 que sólo reacciona con E-cadherinas mutantes (cadherinas que carecen del exón 9), demostró que esta proteína mutante está presente en $13 \%$ de los casos de adenocarcinoma tipo difuso o de células en anillo de sello considerados inmunohistoquímicamente positivos para E-cadherina. De lo dicho anteriormente, se entiende que la invasividad y potencial metastásico de los adenocarcinomas depende de la pérdida de la integridad de todo el complejo de moléculas de adhesión (incluida la E-cadherina), y no sólo eso es necesario al analizar la 
invasividad, sino que también influye la respuesta inmunológica a la neoplasia, como lo plantean Guzmán y cols. (21). En el presente trabajo no pudimos estudiar el grado de invasividad dado que el estudio fue realizado en biopsias endoscópicas, pero recomendamos en un futuro trabajo que la invasividad de los adenocarcinomas gástricos deberá ser evaluada teniendo en cuenta los factores arriba mencionados

La metilación es un mecanismo por el cual los genes pueden ser inactivados, y de esto forma, promover la aparición de un cáncer; en el gen de la E-cadherina CDH1 este mecanismo parece tener mucha importancia. Estudios realizados en China, han demostrado una asociación entre la frecuencia de metilación de $\mathrm{CDH}-1$ e infección por $\mathrm{He}$ licobacter pylori, siendo esta asociación reversible ya que la erradicación de $H$. pylori se asocia a una reducción en la tasa de metilación de E-cadherina $(23,24)$. En nuestro trabajo, de la muestra a la que se estudió E-cadherina, se encontró Helicobacter pylori en sólo 3 casos de los adenocarcinomas tipo intestinal, y en ningún caso de los adenocarcinomas tipo difuso; evidentemente el tamaño muestral limita el estudio de esta interesante asociación.

En conclusión, nuestros datos señalan una mayor expresión de E-cadherina en los carcinomas tipo intestinal en comparación con los carcinomas de células en anillo de sello o difuso.

\section{BIBLIOGRAFÍA}

1. Lauren P. The two histological main types of gastric carcinoma. Diffuse and so-called Intestinal type carcinoma: an attempt at histoclinical classification. Acta Patho Microbiol Scand 1965; 64: 31-49.

2. Ackerman's RJ. Surgical pathology. $9^{\text {th }}$ ed. St. Louis: Mosby; 2004

3. Fuchs CS, Mayer RJ. Gastric carcinoma. N Engl J Med 1995; 333 : 32-41.

4. Mecklin JP, Nordling S, Saario J. Carcinoma of the stomach and its heredity in young patients. Scand J Gastroenterol 1988; 23: 307-11.

5. Lauren PA, Nevalainen JT. Epidemiology of intestinal and diffuse types of gastric carcinoma: a time-trend study in Finland with comparison between studies from high- and low-risk areas. Cancer 1993; 71: 2926-33.

6. Parsonnet J, Vandersteen D, Goates J, Sibley RK, Pritikin J, Chang Y. Helicobacter pylori infection in intestinal- and diffuse-type gastric adenocarcinomas. J Natl Cancer Inst 1991; 83: 640-3.

7. Wright P, Williams G. Molecular biology and gastric carcinoma. Gut 1993; 34: 145-7.

8. Becker KF, Atkinson MJ, Reich U, Becker I, Nekarda EH, Siewert
JR, et al. E-cadherin gene mutations provide clues to diffuse type gastric carcinomas. Cancer Res 1994; 54: 3845-52.

9. Hamilton SR, Aaltonen LA, editors. World Health Organization Classification of Tumours. Pathology and Genetics of tumours of the Digestive System. Lyon: IARC Press; 2000.

10. Batlle E, Sancho E, Francí C, Domínguez D, Monfar M, Baulida J, et al. E-cadherin gene mutations provide clues to diffuse type gastric carcinomas. Nat Cell Biol 2000; 2: 84-9.

11. Humphries MJ, Newham P. The structure of cell-adhesion molecules. Trends Cell Biol 1998; 8: 78-83.

12. Kim HC, Kim HJ, Kim JC. Reduced E-cadherin expression as a cause of distinctive signet-ring cell variant in colorectal carcinoma. J Korean Med Sci 2002; 17: 23-8.

13. Chu PG, Weiss LM. Immunohistochemical characterization of signet-ring cell carcinomas of the stomach, breast, and colon. Am J Clin Pathol 2004; 121: 884-92.

14. Zhang HK, Zhang QM, Zhao TH, Li YY, Yi YF. Expression of mucins and E-cadherin in gastric carcinoma and their clinical significance. World J Gastroenterol 2004; 10: 3044-7.

15. Chen HC, Chu RY, Hsu PN, Hsu PI, Lu JY, Lai KH, et al. Loss of Ecadherin expression correlates with poor differentiation and invasion into adjacent organs in gastric adenocarcinomas. Cancer Lett 2003; 201: 97-106

16. Yong-Ning Z, Cai-Pu X, Biao H, Min L, Liang Q, Dian-Chun F, et al. Expression of E-cadherin and $\beta$-catenin in gastric carcinoma and its correlation with the clinicopathological features and patient survival. World J Gastroenterol 2002; 8: 987-93.

17. Guilford P, Hopkins J, Harraway J, McLeod M, McLeod N, Harawira $\mathrm{P}$, et al. E-cadherin germline mutations in familial gastric cancer. Nature 1998; 392: 402-5

18. Gayther SA, Goringe KL, Ramus SJ, et al. Identification of germ-line E-cadherin mutations in gastric cancer families of European origin. Cancer Res 1998; 58: 4086-9.

19. Ramos de la Medina A, More H, Medina-Franco H, Humar B, Gamboa A, Ortiz LJ, et al. Single nucleotide polymorphisms (SNPs) at CDH1 promoter region in familial gastric cancer. Rev Esp Enferm Dig 2006; 98: 36-41

20. Grötzinger C, Kneifel J, Patschan D, Schnoy N, Anagnostopoulos I, Faiss S, et al. LI-cadherin: a marker of gastric metaplasia and neoplasia Gut 2001; 49: 73-81.

21. Guzmán G, Pablo, Araya O, Juan, Villaseca H, Miguel, et al. Expresión inmunohistoquímica del complejo E-caderina-catenina en cáncer gástrico: Relación con variables clínico-morfológicas y sobrevida de pacientes. Rev Med Chile 2006; 134: 1002-9.

22. Becker KF, Kremmer E, Eulitz M, Becker I, Handschuh G, Schuhmacher $\mathrm{C}$, et al. Analysis of E-cadherin in diffuse-type gastric cancer using a mutation-specific monoclonal antibody. Am J Pathol 1999; 155: $1803-9$.

23. Chan AO, Lam SK, Wong BC, Wong WM, Yuen MF, Yeung YH, et al. Promoter methylation of E-cadherin gene in gastric mucosa associated with Helicobacter pylori infection and in gastric cancer. Gut 2003; 52: 502-6.

24. Leung WK, Man EP, Yu J, Go MY, To KF, Yamaoka Y, et al. Effects of Helicobacter pylori eradication on methylation status of Ecadherin gene in noncancerous stomach. Clin Cancer Res 2006; 12: 3216-21. 